\title{
DYNAMIC ANALYSIS OF A 4-AXLES RAILWAY VEHICLE MODEL
}

The paper deals with using dynamic simulations in fields of construction, production and operating of railway vehicles. It contained process of dynamics solution of choices referent vehicle by the help special solution software - MSC ADAMS. The creating of virtually model included creating model for railway vehicle and the model of track which is assigned for motion of railway vehicle.

In the end of paper there are summary of obtained data from dynamic analysis which give us integrated view of railway vehicle behavior by the run on given track.

\section{Introduction}

Rising demands on construction, used materials and rail vehicle technical equipment also increased the overall complexity, software dependency and last but not least also the total costs of the vehicle production. Currently, vehicle manufacturers focus on vehicle safety, taking into account the overall comfort and operating safety of the vehicle itself and also to provide additional comfort for passengers and service staff, increase the transport capacity while, at the same time minimizing the total and maintenance costs. However, to be able to achieve the afore-mentioned goals, alternative development and production routines must be used in the forthcoming railway vehicles.

Prior the real-life deployment of a new technical solution, we must test all vehicle parameters and properties via practical experiments.

Simulations and analyzes are gaining more and more attention in the process of vehicle production. This allows us to obtain data about vehicle operating behavior from various, sometimes highly extreme conditions before the actual production of a new vehicle is launched. This way we can study effects of input parameters on the operation, safety, service life and reliability of single vehicle components, railway vehicles or complex train sets.

By data evaluation from experimental measurements or from computer simulation we can evaluate railway vehicle dynamics.

\section{Railway Vehicles Simulation Computations}

Simulation computations represent a feasible means of analyzing railway vehicles. In our case they consist of analysis of dynamic properties. By entering relevant input parameters we can obtain vehicle evaluation details pertaining to the vehicle behavior on the track.

It is advantageous that obtaining relevant parameters about vehicle motion we don't have to perform any real-life measurements on the railway vehicle or the actual track. But it is more demanding to obtain correct values of geometric, mass and flexible joint parameters of a mechanical system for creating a mathematic and subsequently a virtual vehicle model. Track parameters are also very important because they can severely influence all our computations.

\subsection{Virtual model}

To create a dynamic model, one must have deep knowledge of mechanical system properties - in our case those pertaining to the model of the railway vehicle. This data analysis is performed on the basis of existing railway vehicles used in practical real-life scenarios. In practice we know that the production vehicle contains a broad spectrum of properties, some of which are more or less needed. These properties are mutually interconnected in a very narrow manner.

Regardless of which vehicle is used, it stands to reason that without sufficient knowledge of the given problem, parameters and an express definition of simulation computations purposes the output will only be a mixture of partial or incomplete results. [2].

\subsection{Computation model}

The computational model can be created on the basis of various points of view but the critical criterion is always the purpose of

\footnotetext{
* Zuzana Ondrova

Department of Traffic and Operating Technique, Faculty of Mechanical Engineering, University of Zilina, E-mail: zuzana.ondrova@fstroj.uniza.sk
} 
the analysis. Models can be viewed in a multitude of manners, including the following representations: geometrical, plane or space models, furthermore as linear or nonlinear models and also according to the type of analyzed field (e. g. frequency, time domain etc.)

The computation model used in my work is a nonlinear model whereby the bogie frame and car body are rigid and interconnected with elastic joints. Dynamic simulation was carried out in the time domain.

Solution of mathematical model is based on finding all characteristic numbers and characteristic vectors. The majority of used mathematic methods are assigned to the solution of characteristic number standard problem, meaning that the general problem must first be transformed into a standard problem.

Of the available classic solution methods, the Jacob's method of rotation is very convenient in our case. However, other methods, such as Rayleigh-Ritz method, method of inverse iteration, GrammSchmidt orthogonal method, Lanczo's method etc. may also be used. [2].

\section{Programs for Simulation Computations}

Current computer assistance program products include a range of intelligent tools and software modules which enhance the efficiency of the engineer. These systems analyze possible solutions and reject those which are unsuitable due to restrictions imposed by physical laws or restrictions defined within the project.

The MBS (Multi Body Simulation) technology is most commonly used in solution and evaluation of various dynamic properties of mechanical systems. It is based on simulation of mechanical systems (MSS) and the subsequent definition of local stresses in every instant of time. MBS offers simple simulation of active components such as shafts, crank shafts etc. In some cases it is possible to use history of loading results obtained by MBS when solving real-life mechanism by FEM (finite element method).

MBS has one great advantage - it allows the evaluation of various effects that influence the system. In the case of railway vehicles, it is possible to detect component fatigue depending on suspension settings, operation style or track quality.

\subsection{Aplications - ADAMS/Rail}

Dynamic analysis problems can be solved using software specifically designed for the field of railway vehicles. The Rail module is intended for simulation computations of railway vehicles. This module performs dynamic simulations of mechanical system parameters not only with arbitrary structures but also for a combination of rigid and flexible bodies upon which inertia, gravity and excitation forces are exerted.

To assure proper functioning of all stages of railway vehicle development, testing, production, construction and operation, the program must be interfaced with all leading producers to allow data sharing and data interchange.

The option to create an animated model showing the actual movement of the object and a graphical description of all physical parameters such as speed, acceleration, shift, forces, etc. are all useful extensions of this program. This module also includes the computation of mechanical system characteristic frequency and also various pathways of vehicle excitation.

It is important to create a model that is as accurate as possible - otherwise simulation results will be unpredictable. Thus, we must know how to prepare input parameters and how to use them to achieve our goal purpose.

In addition to the previous, the vehicle model must be appropriately combined with the track model to assure complete evaluation during dynamic simulation and to mimic the railway vehicle behavior when running on tracks [1].

\section{Dynamic Analysis of a 4-axle two - Bogies Railway Vehicle Model}

The reference vehicle used in the dynamic analysis is a 4-axle two - bogies coach wagon that is otherwise known as ERRI Wagon. The vehicle model is equipped with springs and dampeners with nonlinear characteristics. Geometric parameters, mass parameters and parameters of elastic joints are identical to those present in the simulated model in MSC ADAMS/Rail.

Wheels of the wheelset have a wheel profile of S1002. The axle guiding in bogie frame is supported by a bail arm. Wheelsets are connected to the bogie frame spring via primary suspension. This system consists of a coil spring and hydraulic dampers located between the axle box and the bogie frame. The car body is connected to the bogie frame spring via a system of secondary suspensions [2].

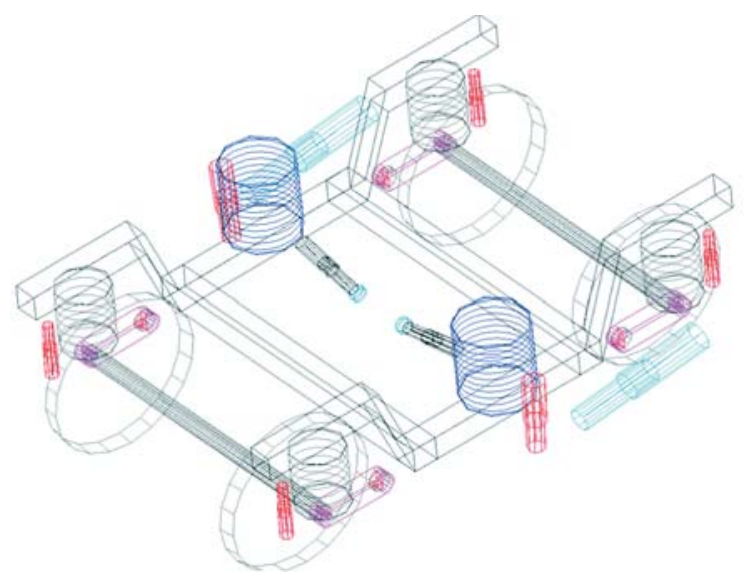

Fig. 1 Bogie frame model (ADAMS/Rail) 


\subsection{Vehicle model}

The vehicle model consists of the bogie frame (bogie subsystem) and a car body (subsystem of car body) model. Each subsystem assembly contains several construction components, in addition to the car body model which consists of one complex unit. These parts have properties and geometric parameters based on real-life railway vehicles.

The assembly of the ERRI model is comprised of the following three subsystems:

- car body

- front bogie

- rear bogie.

To assemble the model, all components (wheelset, bogie frame, suspension (primary and secondary), dampers (lateral, vertical and anti-yaw dampers) etc.) must be defined. Pre-existing templates can also be modified according to our own requirements by simply changing the model parameters [1].

Each simulation/analysis takes into account real-life data. If the desired requirements are not met, it is necessary to change the geometric form of the model or to add data otherwise neglected at the stage of model reduction (additional friction, definition of components elasticity which are not rigid, etc.). The simulation can be rerun several times until goal output parameters are met.

\subsection{Track model}

The track model is based upon real-life geometrical data, specifically the track is located between SURANY - ULANY NAD
ZITAVOU. I chose this track on the basis of data availability. This track is representative of dynamic analysis due to the presence of many curves with various radius parameters and the resulting sections of transit curves and ascending rails, important for general evaluation of vehicle behavior during operation on the given track.

The chosen track has a length of $5882 \mathrm{~m}$ and there are five right and a three left-hand curves. The complete track data was imported into the simulation software (all curve radiuses, degree of rail incline and track leveling). Fig. 4 shows the track overview. The track radius and particular lengths of total track length are displayed in Fig. 4. The maximum vehicle speed allowed on the whole track is $60 \mathrm{~km} / \mathrm{h}$. All my computations were computed at this speed. Vehicle derailment protection was analyzed and compared for variants of rail profiles and cant rail UIC60/1:40, S49/1:40 and $\mathrm{S} 49 / 1: 20$ [2].

\section{Vehicle Derailment Protection}

Vehicle derailment protection is defined as the ratio between the guide force and the wheel force. Results which are presented in Fig. 6 represent a variation of forces in wheel/rail interaction.

To simplify the orientations in these results we can state that:

- The bottom part of the graph describes the track geometry.

- The vertical axis on the left-hand side of graph specifies the reciprocal of curve radius,

- The vertical axis on the right-hand side of graph specifies the degree of incline value of outer stretch of rails.

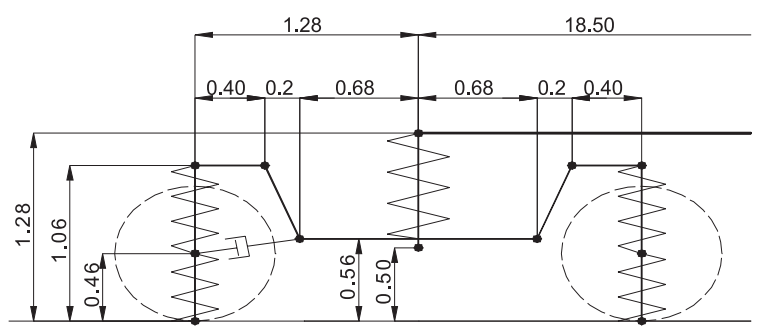

Fig. 2 Geometric parameters of vehicle model [2]

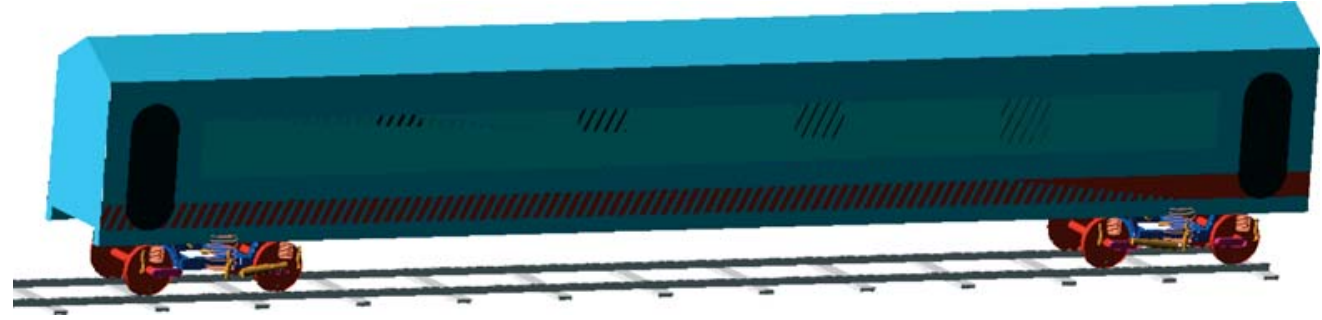

Fig. 3 Vehicle model ERRI (ADAMS/Rail) 


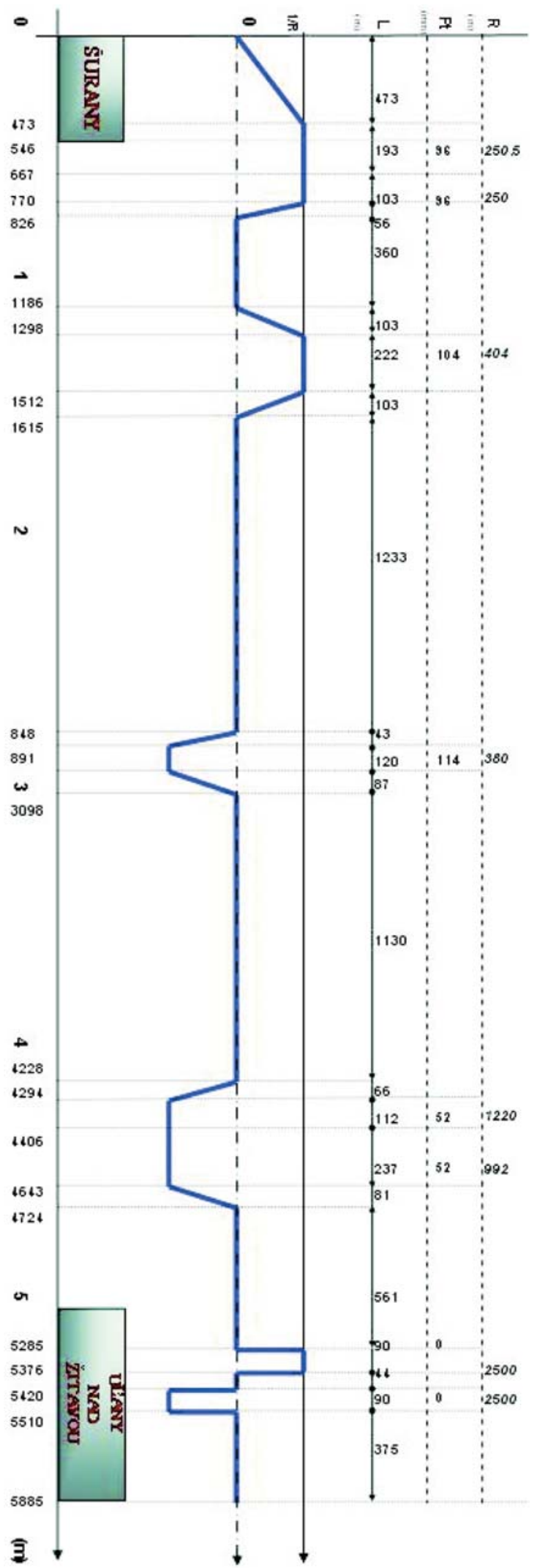

Fig. 4 Track overview diagram [2]
- The upper half and positive values of radius are characteristic of right-hand curves, the lower half and negative values of radius are characteristic of left-hand curves.

- The degrees of incline are numbered upwardly from zero (from below).

In the other graphs there are descriptions of wheel forces $(\mathrm{Q})$ in $[\mathrm{N}]$, guide forces $\mathrm{Y}[\mathrm{N}]$ and vehicle derailment protection (BPV), which have a non-dimensional value [-]. Identification of single positions on vehicle bogies is in conformance with marked output (Fig. 5) signal and can be obtained at the end of the simulation computations [2].

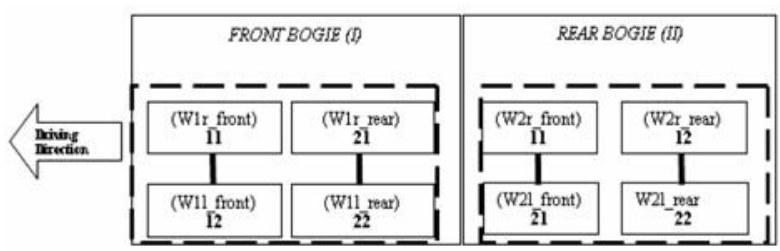

Fig. 5 Location of positions in vehicle [2]

\section{Conclusion}

From Fig. 6 it is evident that the majority of the load is exerted onto the wheel during curve cornering - which is the thread up wheel of the first wheelset from the first bogie of vehicle. The right guide force creation and transmission in the horizontal plane is located within this point. The second wheel of the thread up wheelset is assigned to decrease the wheel and also guide force. Influence of transient performance in the force range can be seen in the transit curve. On locally straight tracks the wheel force nominal values are stable and the values of guide forces are close to zero.

Variation of forces confirmed the running stability of our vehicle and the relationship between the vehicle virtual model and the track is comparable to the expected real-life conditions.

The results of wheel and guide forces as well as vehicle derailment protection are all within the allowed limits. The worst ratio of vehicle derailment protection on this section is 0.5 , which is less than the permissible value of 1.2 for wheel profile S1002.

The created vehicle model can be used in other simulations by simply changing some of the parameters of the vehicle and can also be employed when evaluating passenger comfort if various acceleration forces that act upon the vehicle are obtained. 

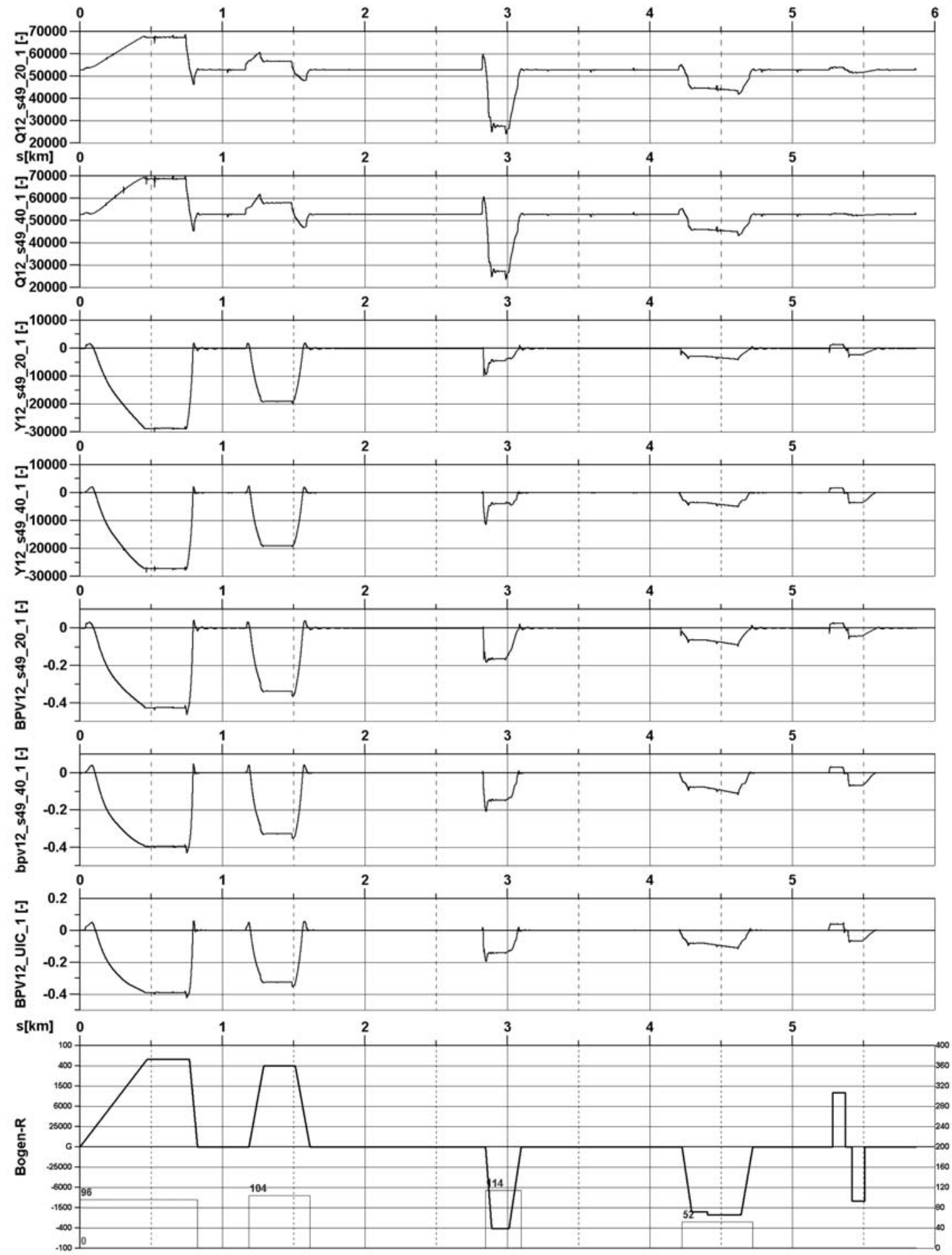

Fig. 6 Evaluation of chosen parameters for rail profile S49 and cant rail / 1:20 a 1/40 and UIC [2]

\section{References}

[1] ONDROVA, Z., GERLICI, J., LACK, T.: Analysis of Dynamical Properties for Rail Vehicle at Travel wake of Real Track (in Slovak), SETRAS 2006, ISBN 80-969165-8-0. pp. 163-169. EDIS - University of Zilina, 2006.

[2] ONDROVA, Z. (2008): Dynamical Simulations of Travel Rail Vehicel wake of Real Track (in Slovak), Dissertation thesis, 2008. 